\title{
Probabilistic Power Flow Model to Study Uncertainty in Power System Network Based Upon Point Estimation Method
}

\author{
Li Bin', Muhammad Shahzad', Qi Bing1, Muhammad Raza Zafar², Rabiul Islam³, \\ Muhammad Umair Shoukat ${ }^{4}$
}

${ }^{1}$ School of Electrical and Electronic Engineering, North China Electric Power University, Changping District, Beijing, China

${ }^{2} \mathrm{PMU}, \mathrm{MEPCO}$, Multan, Pakistan

${ }^{3}$ Sugar \& Food Industries Corporation, Dhaka, Bangladesh

${ }^{4}$ Department of Electrical Engineering, Government College University Faisalabad Sahiwal Campus, Sahiwal, Pakistan

\section{Email address:}

direfish@163.com (Li Bin), shahzadpansota@hotmail.com (Muhammad Shahzad), bellqi@yeah.net (Qi Bing), musapmu2009@hotmail.com (M. R. Zafar),1657345663@qq.com (R. Islam), umairshoukat@yahoo.com (M. U. Shoukat)

\section{To cite this article:}

Li Bin, Muhammad Shahzad, Qi Bing, Muhammad Raza Zafar, Rabiul Islam, Muhammad Umair Shoukat. Probabilistic Power Flow Model to Study Uncertainty in Power System Network Based Upon Point Estimation Method. American Journal of Electrical Power and Energy Systems. Vol. 6, No. 5, 2017, pp. 64-71. doi: 10.11648/j.epes.20170605.11

Received: July 18, 2017; Accepted: July 31, 2017; Published: August 22, 2017

\begin{abstract}
In this paper, point estimation approach is used to calculate the statistical moment of a random quantity that is a function of $m$ input random variables. In this work, loads of the proposed network is considered as a random variable. Two special cases of point estimation approach are considered such as $2 m$ and $2 m+1$ concentration schemes. In $2 m$ concentration scheme, skewness is considered, but in $2 m+1$ concentration scheme, both skewness and kurtosis are taken into account for probability density function. The proposed model is investigated using P. M. Anderson 9-bus test system. As a result, by changing the value of a random variable that follows a predefined distribution, expected bus voltage magnitude and expected line loading are identified. For the comparison purpose, $2 m$ and $2 m+1$ scheme was compared with deterministic load flow analysis.
\end{abstract}

Keywords: Probabilistic Power Flow, Point Estimation Method, Random Variable, Uncertainty Analysis

\section{Introduction}

Power flow study in power system is an important tool. It is mostly used to evaluate the voltage magnitude and angle at different buses in power system, active and reactive power flow through the line. For the expansion, operational planning, real-time operation, and control of power system; power flow has become a key. As the power system becoming more and more complex, several problems such as power system planning, operation, and control are a challenge for engineers. In order to tackle said problem, different studies are carried out in [1], [2].

Due to the integration of renewable energy in power system network, it is becoming more sensitive to above problems. The new challenges for the network are voltage
Stability and transient stability. Although, different types of changes are noticed in recently published papers [3], [4]. Among of these problems, uncertainty in renewable sources is a major challenge for engineers. These uncertainties are unpredictable in a renewable generation such as the wind and solar energy, daily load variation, inherent randomness of fault, and failure of the power system.

A classical way of power flow analysis is a deterministic power flow (DPF) analysis. DPF analysis has not the capability to handle the future problems. DPF analysis only calculates the values chosen by the analyst [5]. You can say that DPF analysis provides the variable values in term of certain load and generation characteristics. Its accuracy depends on the input data provided by the analyst. The generation pattern, future load characteristic, and faults in power system network become more unpredictable nowadays. 
In DPL analysis, it is impractical to access the probabilistic power flow analysis. For each possible computation need to perform the analysis separately that is not only time consuming but also hectic. To handle the said problem, probabilistic power flow (PPF) analysis is taken into consideration. PPF analysis has the capability to handle almost all kind of uncertainties occurs in power system network. A few decay ago, PPL analysis has become a hot research topic as shown [6], [7], [8], [9], [10] recent publication.

PPF analysis of power system can be categories into three approaches such as simulation approach, analytical approach, and approximate approach. In simulation approach, Monte Carlo simulation (MCS) is very famous and had been used in different papers [11], [12], [13]. In this method, a certain probability distribution is assigned to a certain variable. A random number is selected from the probability distribution, and computation is performed. In this approach, to attain the more precise results, need a large number of iteration that is the result of extensive computation time and storage, which are drawbacks of this approach. To overcome this computational time analytical approach was proposed in [14], [15], [16]. In analytical approach linearization of power flow equation is the main drawback, so that it can work with probability density function.

In approximate practices, no need to linearization the power flow equation, it gives the approximate statistical information about the output random variables. By using several techniques, the evaluation point can be decreased. In this way computation time and storage can be considerably reduced. In approximate techniques, Point Estimation Method (PEM) can reduce the considerable computation time as well as a precise result. PEM calculate the statistical moments of a random variable that is a function of input random variable.

Probabilistic PEM is proposed in this paper. Although, a number of research papers have been published at this method e.g. probabilistic optimal power flow in electricity market based on the $2 m$ scheme is proposed in [17], and a probabilistic load flow based on nonparametric density estimator is proposed in [18] but in the above research papers $2 m$ and $2 m+1$ concentration scheme are used separately.

In this paper, probabilistic power flow model is proposed with point estimation method, two special cases $2 m$ and $2 m+1$ concentration schemes are used, a total load of the proposed network is considered as a mean value, and an arbitrary value of standard deviation is selected for creating the uncertainty in load. In this work, few statistical moments (i.e. mean, variance, skewness, and kurtosis) are carried out to determine the effect of uncertainty on network parameters. Especially, by varying the value of skewness and kurtosis different results are observed. These results can be used for future planning, corrective action, and operation of electric power distribution system. The rest of the paper is organised as follow; Section 2 provides an introduction of PEM and corresponding schemes. Section 3 provides the information about test system. The simulation results and discussion is presented in Section 4. Finally, Section 5 conclude the paper.

\section{Point Estimation Method}

The main categorization of probability and statistic is as,

(I) Descriptive statistic

(II) Probability statistic

(III) Inferential statistic

In inferential statistic, it is used to draw a conclusion about a given population information about a representative in a decision making. It is impractical, expensive, and impossible to measure the large population, by using the samples, it can easily estimate, predict, generalise, and make a decision about large population. Two main methods are employed in this case i.e. point estimator and maximum likelihood method. Here, point estimate method has been considered.

The first PEM was proposed in 1975 by Rosenblueth, at that time, it was used for symmetric variables. It was modified in 1981 for asymmetric variables. Since that, a different method was used to improve the origin Rosenbluth method, but the main difference is to change the types of random variables that they used to improve the performance.

In this method, the sample is used to estimate corresponding population parameter desirable characteristic. Further, classification of this method depends on the selection of estimator value; it may be an unbiased estimator, consistence estimator, and maximum likelihood estimator. In this paper unbiased estimator is selected for analysis.

In unbiased estimator, the mean of the sample distribution should be the same as the target parameter. Simply, the sample mean is an unbiased estimator for the population mean. Variance and minimal variance of the sample is also considered as an unbiased estimator of population variance but in this work mean is consider as an unbiased estimator because mean is the best possible estimator for the population mean if information is given in the sample.

The aim of point estimator is to calculate the statistical moment of the random quantity that is a function of one or more input random variables. Let $Z$ denote a random quantity that is a function of $X$ input random variables. Then, evaluate the random quantity $Z=F(X)$, to estimating the mean value of $Z$.

\subsection{Formulation of PEM}

Let $p_{l}$ be an input random variable having a density function (DF) $f_{p l}, F$ is function that relate the input and output variable information about the uncertainty problems. This method concentrate the information provided by the first few central moment of input random variable. $K$ is the number of points on the input random variable called concentration. By using $k$ and $F$ information, uncertainties associated with output random variables are calculated. The concentration of input random variable $k$ is compose of two parameter (i.e. $p_{l, k}, \omega_{l, k}$ ). Here, $p_{l, k}$ is the $k t h$ value of $p_{l}$, it is a location parameter and $\omega_{l, k}$ is weighting factor, both contribute in the output random variable results. The number of $k$ evaluation is depends upon the selection of concentration scheme. Total number of evaluation of $F$ is $k \times m$, here, $\mathrm{m}$ is 
number of input variables. If $2 m$ scheme is selected then $k \times m$ will be the evaluation points. If $2 m+1$ scheme is selected then $k \times m+1$ will be the evaluation points. The input vector form input random variables $m$ with mean is,

$$
Z(l, k)=F_{i}\left(\mu_{p 1}, \mu_{p 2, \ldots \ldots . .,} \mu_{p l, \ldots \ldots \ldots . . . .} \mu_{p m}\right)
$$

$K_{t h}$ Concentration of input random variable is evaluated by using statistical input data. The location parameter is calculated by using [22], that is:

$$
p_{l, k}=\mu_{p l}+\xi_{l, k} \sigma_{p l}
$$

Here,

$p_{l, k}=$ location parameter of input random variable

$\mu_{p l}=$ mean of input random variable

$\xi_{l, k}=$ standard location of input random variable

$\sigma_{p l}=$ standard deviation of input random variable

Here, $\xi_{l, k}$ depending upon the type of concentration scheme, in this paper two concentration scheme are considered.

\section{2. $2 m$ Concentration Scheme}

In this scheme, standard location of input random variable is calculated by using below equation [22]:

$$
\xi_{l, k}=\frac{\lambda_{l, 3}}{2}+(-1)^{3-k} \sqrt{m+\left(\lambda_{l, 3} / 2\right)^{2}} k=1,2
$$

Here, $\xi_{l, k}$ depend upon the number of input random variables, from the Eq. (2), it is clearly shown that as the $m$ increase location of $p_{l}$ move away from the mean $\mu_{p l}$ according to $\sqrt{m}$. In Eq. (3) parameter $\lambda_{l, 3}$ denoted the skewness of input random variable that is computed as:

$$
\lambda_{l, 3}=\frac{E\left[\left(p_{l}-\mu_{p l}\right)^{3}\right]}{\left(\sigma_{p l}\right)^{3}}
$$

The weighting of the concentration located at Eq. (1), then used to estimates to take into account the skewness of probability distribution of $Z$. the value of $\omega_{l, k}$ is range from 0 to 1 and sum of all values of $\omega_{l, k}$ is unity.

$$
\omega_{l, 1}=\frac{-1}{m} * \frac{\xi_{l, 2}}{\left(\xi_{l, 1}-\xi_{l, 2}\right)}, \omega_{l, 2}=\frac{1}{m} * \frac{\xi_{l, 1}}{\left(\xi_{1,1}-\xi_{l, 2}\right)}
$$

The advantage of the $2 m$ scheme is related to its simplicity, lesser computation burden, and the real value of concentration.

\section{3. $2 m+1$ Concentration Scheme}

In this scheme, three points are taken from each input random variable and one location fixed for mean value. Four statistical moments are carried out for PDF of random variables. Standard location of $k$ concentration can be found by using [19], [20], [21] below equation.

$$
\begin{aligned}
& \xi_{l, k}=\frac{\lambda_{l, 3}}{2}+(-1)^{3-k} \sqrt{\lambda_{l, 4}-\frac{3}{4} *\left(\lambda_{l, 3}\right)^{2}} \\
& \mathrm{~K}=1,2 \& \xi_{l, 3}=0
\end{aligned}
$$

The weights are calculated as:

$$
\begin{aligned}
\omega_{l, k} & =\frac{(-1)^{3-k}}{\xi_{l, k}\left(\xi_{l, 1}-\xi_{l, 2}\right)} \mathrm{k}=1,2 \& \\
\xi_{l, 3} & =\frac{1}{m}-\frac{1}{\left(\lambda_{l, 4}-\lambda_{l, 4}{ }^{2}\right)}
\end{aligned}
$$

From the Eq. (7), $\lambda_{l, 4}$ is kurtosis taken into account that's why $2 m+1$ scheme is more accurate then $2 m$ scheme but 1 additional evaluation is needed.

In this method, after estimating the sample point, the fitness function is calculated for all estimated points. The uncertainty is transfer from the input random variables to the output random variables by using $F$ function, and $Z(l, k)$ is the vector of the output random variables associated with the $K$ th concentration of random variable $p_{l}$. The total number of deterministic analyses to be run depends on the concentration scheme considered. Finally, the expected results is computed as:

$$
E|Z|=\sum_{l=1}^{m} \cdot \sum_{k=1}^{K}\left(\omega_{l, k}\right) * Z(l, k)
$$

Here, output random variable is $\boldsymbol{Z}$ and $E|Z|$ is the expected value of vector $\boldsymbol{Z}$, input random variable, and the number of points is denoted by $m$ and $k$ respectively.

\subsection{Skewness}

It measures the asymmetry of the distribution data of the random variable about its mean. It is a third central moment of distribution, three different value of the coefficient is used for analysis. Its value may be positive, negative, and even undefined. Fisher Pearson gave an excellent concept about skewness. He gave the constant value of each distribution, by using (9). In this work, random variable follows the normal distribution.

$$
K S=\frac{\sum_{i=1}^{n}\left(X_{i}-X^{-}\right)^{3} / N}{\sigma^{3}}
$$

Here, $N$ is used rather than $N-1$, for calculating the standard deviation.

$K s=$ coefficient of skewness

For a normal distribution, if $K s=0$, it is symmetrical distribution, if $K s<0$, it mean data distribution is negatively skewed, if $K s>0$, it means data is positively skewed.

\subsection{Kurtosis}

It is a fourth central moment of distribution data set. It measures the peak of distribution. Three different value of the coefficient of kurtosis is used for analysis purpose. Pearson explained that value of kurtosis coefficient is equal to 3 in the case of normal distribution, its formula is given below:

$$
K_{K}=\frac{\sum_{i=1}^{n}\left(X_{i}-X^{-}\right)^{4} / N}{\sigma^{4}}
$$

Here, $K_{K}$ is coefficient of kurtosis, when, $K_{K}=3$, it is called Mesohurtic, when the value of kurtosis coefficient is more than 3, this is called Leptokurtic. Leptokurtic 
distribution mean that data is distributed around the mean value. When the value of kurtosis coefficient is less than 3 , it is called Platykurtic distribution. Platyhurtic distribution mean that data is distributed far from the mean value of distribution.

\subsection{Flow Chart for PEM}

In this section, step-by-step implementation of PEM is presented.

Step 1: First of all, set the parameter such as a probabilistic characteristic of an input random variable (i.e. mean, standard deviation, skewness, and kurtosis) and the number of estimation points $k$ is determined. In this paper, $2 m$ and $2 m+1$ concentration schemes are used. So, 2 points in case of the $2 m$ scheme and 3 points in case of the $2 m+1$ scheme are generated. In this paper, a random variable is a total system load that follows a normal distribution and considered one variable $(m=1)$.

Step 2: In this step, standard central moment and standard location $\lambda_{l, k}$ and $\xi_{l, k}$ are calculated respectively corresponding to selected scheme. Here, $\lambda_{l, 1}, \lambda_{l, 2}, \lambda_{l, 3}, \lambda_{l, 4}$ are the mean, standard deviation, skewness, and kurtosis respectively.

Step 3: In this step, input vector for power flow is calculated by using the Eq. (1).

Step 4: In this step, the fitness function is evaluated by the DFL analysis. DFL is derived from the input vector. If the algorithm reached maximum input random variable, it will stop. Otherwise, it follows the step 3.

Step 5: In this step, the algorithm checks the number of point $K$ if it is reached to a maximum number of point it will stop. Otherwise, it follows the step 3.

\section{Flow Chart of PEM}

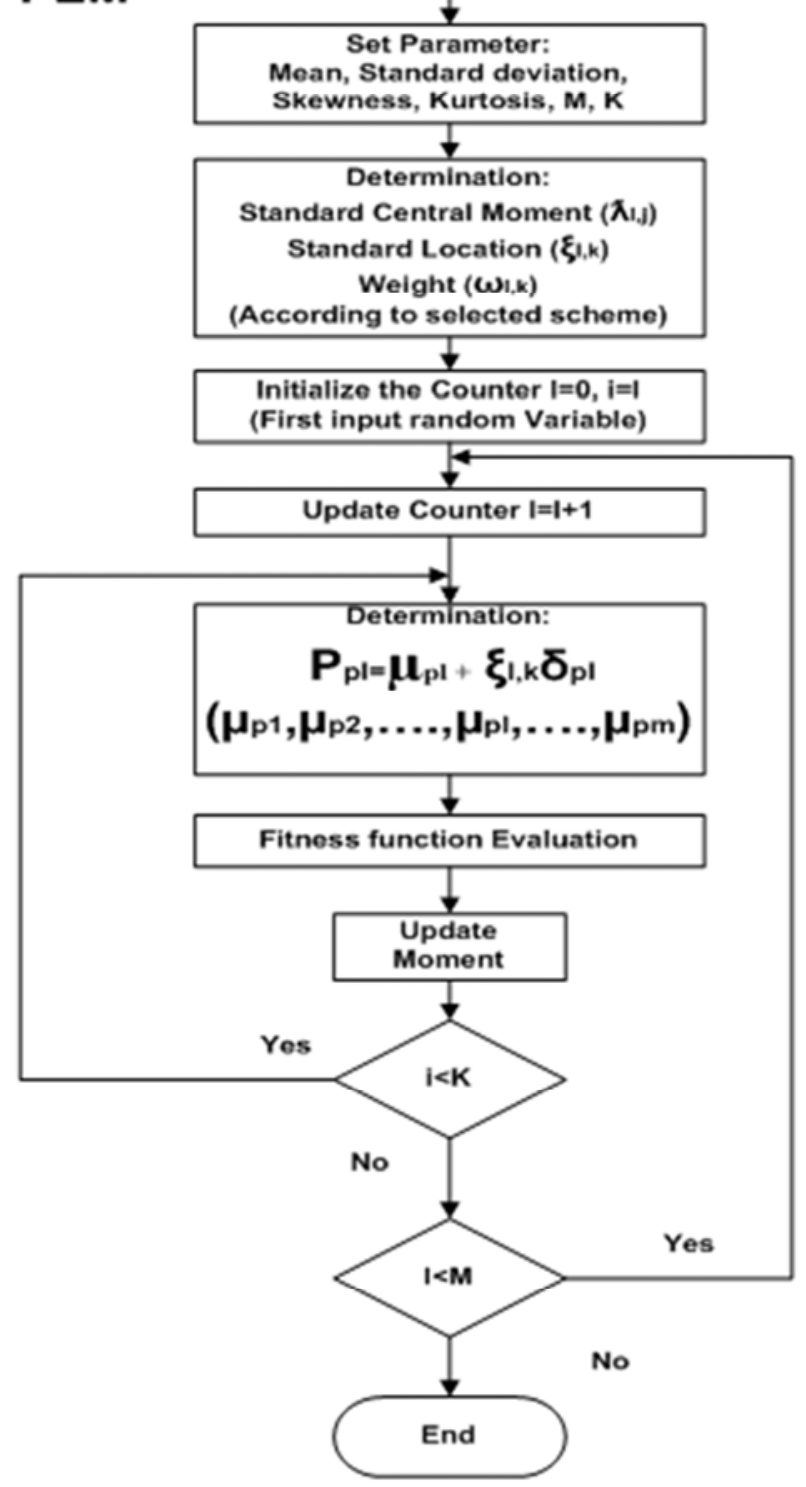

Figure 1. Flowchart of PEM. 


\section{P. M. Anders on 9-Bus Test System}

For the results demonstration purpose, P. M. Anderson 9bus test system is used. All related data to this test system is available in [22], [23]. The total load for base case is 315 MW and 115 Mvar, bus 1 was considered as a reference bus. Some information related to load and line data is given in Tables $1 \& 2$ respectively.

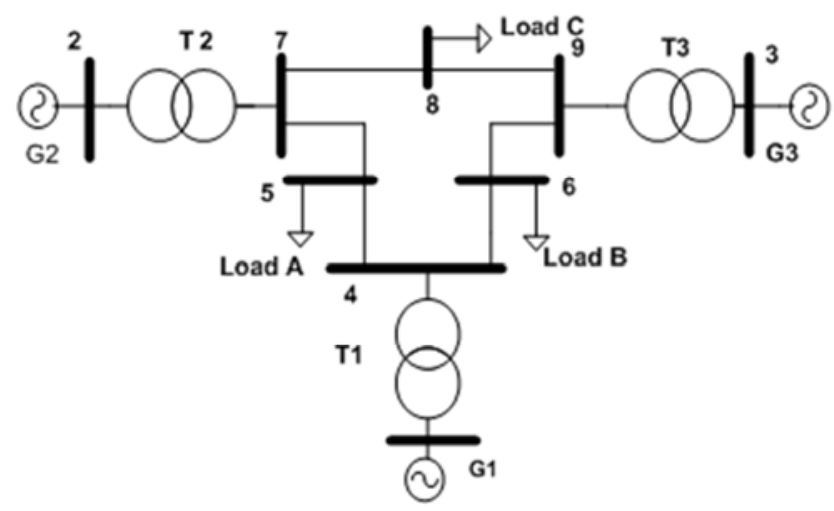

Figure 2. P. M. Anderson 9-bus test system.

\section{Results and Discussion}

In order to verify the accuracy and efficiency of the proposed model, P. M. Anderson 9-bus test system was simulated through DIgSILENT Power Factory software 15.1. In this test system, all PV buses voltage magnitude are set 1.05 (p.u.), and balance Ac load flow analysis was implemented. All results for base case are presented in Tables 2 and 3.

\section{1. $2 m$ Concentration Scheme}

In $2 \mathrm{~m}$ point concentration scheme, the standard deviation is considered as an arbitrary parameter. Three different values of coefficient of skewness are set on $0,0.5$, and -0.5 , its explanation is presented in Section 2.4. Firstly, standard location, weighting coefficient, Active, and Reactive power will be calculated; these parameter values depend upon the selection of standard deviation and the value of the coefficient of skewness. In this study, when standard deviation was selected $10 \%$ and coefficient of skewness 0 . The value of standard location and a weighting coefficient for both point was $(1 \&-1)$ and $(0.5 \& 0.5)$ respectively, then voltage magnitude at each point was calculated (see Fig. 3a). When $k_{s}=0.5$, the value of standard location and a weighting coefficient for both point was $(1.368 \&-0.868)$ and $(0.326 \&$ $0.515)$ respectively, then voltage magnitude at each point was calculated (see Fig. $3 b$ ). Similarly, when $K_{s}=-0.5$, the value of standard location and a weighting coefficient for both point was $(0.868 \&-1.368)$ and $(0.515 \& 0.326)$ respectively, then voltage magnitude at each point was calculated (see Fig. $3 \mathrm{c}$ ). From the above analysis, it is clearly shown that as the value of the coefficient of skewness was increased, the magnitude of active and reactive power injection to the buses also increased. This claim can be confirmed by the Table 4 .

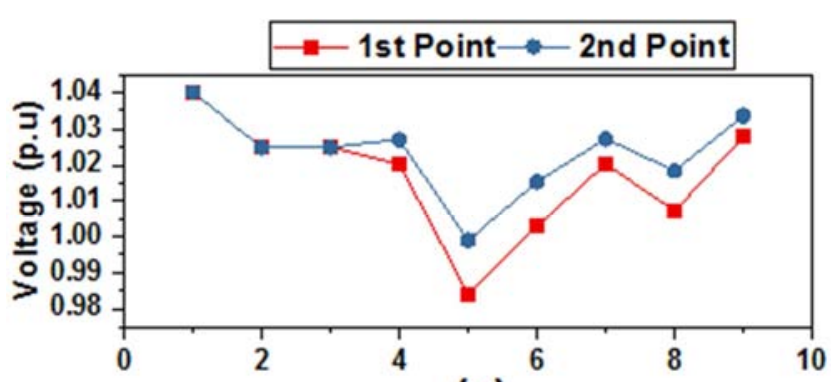

(a)
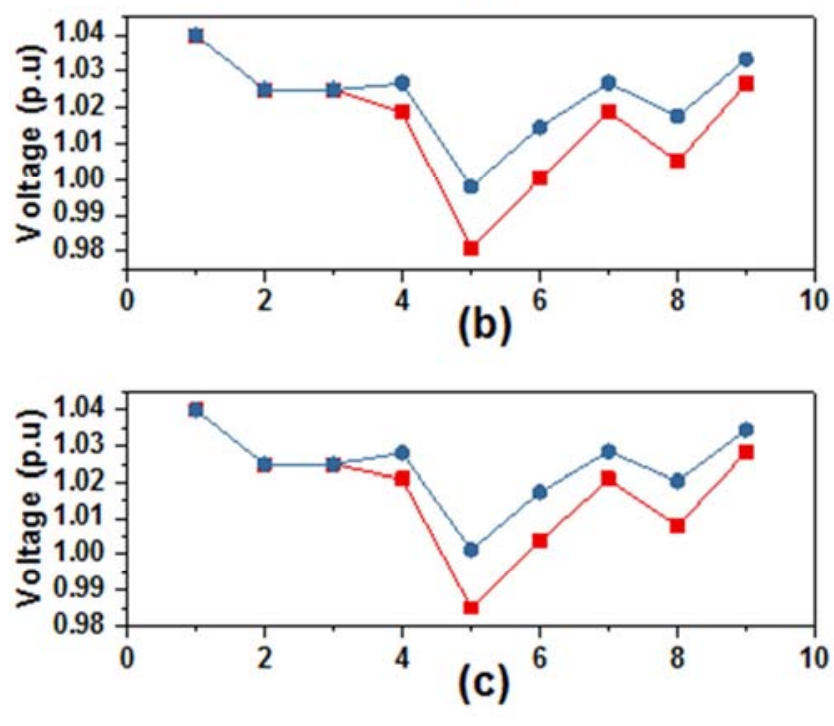

Bus No

Figure 3. Voltage magnitude at each bus, when $K s=0,0.5, \&-0.5$.

Table 1. Base case load data.

\begin{tabular}{ll}
\hline Loads & Load (MVA) \\
\hline Load A & $125+\mathrm{j} 50$ \\
Load B & $90+\mathrm{j} 30$ \\
Load C & $100+\mathrm{j} 35$ \\
\hline
\end{tabular}

Table 2. Base Case voltage magnitude.

\begin{tabular}{ll}
\hline Bus Number & Voltage Magnitude (p.u.) \\
\hline Bus 01 & 1.040 \\
Bus 02 & 1.025 \\
Bus 03 & 1.025 \\
Bus 04 & 1.024 \\
Bus 05 & 0.991 \\
Bus 06 & 1.009 \\
Bus 07 & 1.024 \\
Bus 08 & 1.013 \\
Bus 09 & 1.030 \\
\hline
\end{tabular}

Table 3. Base Case Line Loading.

\begin{tabular}{ll}
\hline Line Number & Loading (\%) \\
Line 01 & 16.160 \\
Line 02 & 20.943 \\
Line 03 & 19.699 \\
Line 04 & 09.129 \\
Line 05 & 14.700 \\
Line 06 & 10.331 \\
\hline
\end{tabular}


Table 4. Active and Reactive Power of two points.

\begin{tabular}{llll}
\hline Skewness $\left(\mathbf{K}_{\mathbf{s}}\right)$ & Points No & $\begin{array}{l}\text { Active Power } \\
\text { (MW) }\end{array}$ & $\begin{array}{l}\text { Reactive Power } \\
\text { (Mvar) }\end{array}$ \\
\hline 0 & 1 & 365.539 & 133.450 \\
& 2 & 299.077 & 109.187 \\
0.5 & 1 & 377.769 & 137.915 \\
& 2 & 303.463 & 110.788 \\
-0.5 & 1 & 361.154 & 131.849 \\
& 2 & 286.847 & 104.722 \\
\hline
\end{tabular}

\section{2. $2 m+1$ Concentration Scheme}

In this scheme, arbitrary parameters such as standard deviation, skewness, and kurtosis $\left(k_{k}\right)$ are set at $10 \%, 0.5$, and 2.5 respectively. The simulation was carried out for three different value of the coefficient of kurtosis such as $2.5,3$, and 3.5 respectively. Its explanation is provided in Section 2.5. Firstly, standard location and weighting coefficient were calculated by using Eq. ( $3 \& 4$ ) respectively. When $k_{k}=2.5$, the value of standard location and weighting coefficient related to three point was $(1.723,-1.419 \& 0)$ and $(0.184,0.224 \& 0.698)$ calculated respectively, then voltage magnitude at each point was calculated (see Fig. 4a). The voltage magnitude When $K_{k}=$ 3 , the value of standard location and weighting coefficient related to three points was $(1.863,-1.559 \& 0)$ and $(0.156,0.187$ $\& 0.759)$ calculated respectively, then voltage magnitude at each point was calculated (see Fig. 4b). When $K_{k}=3.5$, the value of standard location and weighting coefficient related to three points was $(2.004,-1.700 \& 0)$ and $(0.134,0.158 \& 0.806)$ calculated respectively, then voltage magnitude at each point was calculated (see Fig. 4c). From the above analysis, it is clearly shown that, as the value of the coefficient of kurtosis was increased, the load injection in buses are increased. This claim can be confirmed by the Table 5 .

Table 5. Active and Reactive Power of three points.

\begin{tabular}{llll}
\hline kurtosis $\left(\mathbf{k}_{\mathbf{k}}\right)$ & Point No & $\begin{array}{l}\text { Active Power } \\
(\mathbf{M W})\end{array}$ & $\begin{array}{l}\text { Reactive Power } \\
\text { (Mvar) }\end{array}$ \\
\hline \multirow{3}{*}{2.5} & 1 & 389.585 & 142.229 \\
& 2 & 285.136 & 104.097 \\
3 & 3 & 332.308 & 121.319 \\
& 1 & 394.249 & 143.932 \\
& 2 & 280.473 & 102.394 \\
3.5 & 3 & 332.308 & 121.319 \\
& 1 & 398.911 & 145.634 \\
& 2 & 275.811 & 100.692 \\
& 3 & 332.308 & 121.319 \\
\hline
\end{tabular}

Table 6. Camparrision of EVM, when $K s=0 \& k_{k}=3$.

\begin{tabular}{llllll}
\hline Bus \# & $\begin{array}{l}\text { Base } \\
\text { Case }\end{array}$ & $\begin{array}{l}\mathbf{2 m} \\
\text { scheme }\end{array}$ & $\begin{array}{l}\mathbf{2 m + 1} \\
\text { scheme }\end{array}$ & $\begin{array}{l}\text { SD* } \mathbf{2 m} \boldsymbol{\&} \\
\text { Base }\end{array}$ & $\begin{array}{l}\text { SD* 2m+1 \& } \\
\text { Base }\end{array}$ \\
\hline 1 & 1.04 & 1.56 & 1.422 & 0.26 & 0.191 \\
2 & 1.025 & 1.537 & 1.401 & 0.256 & 0.188 \\
3 & 1.025 & 1.537 & 1.401 & 0.256 & 0.188 \\
4 & 1.024 & 1.535 & 1.399 & 0.255 & 0.187 \\
5 & 0.991 & 1.487 & 1.354 & 0.247 & 0.181 \\
6 & 1.009 & 1.513 & 1.379 & 0.252 & 0.184 \\
7 & 1.024 & 1.535 & 1.399 & 0.255 & 0.187 \\
8 & 1.013 & 1.519 & 1.384 & 0.253 & 0.185 \\
9 & 1.030 & 1.546 & 1.409 & 0.257 & 0.189 \\
\hline
\end{tabular}

$\mathrm{SD}^{*}=$ standard deviation
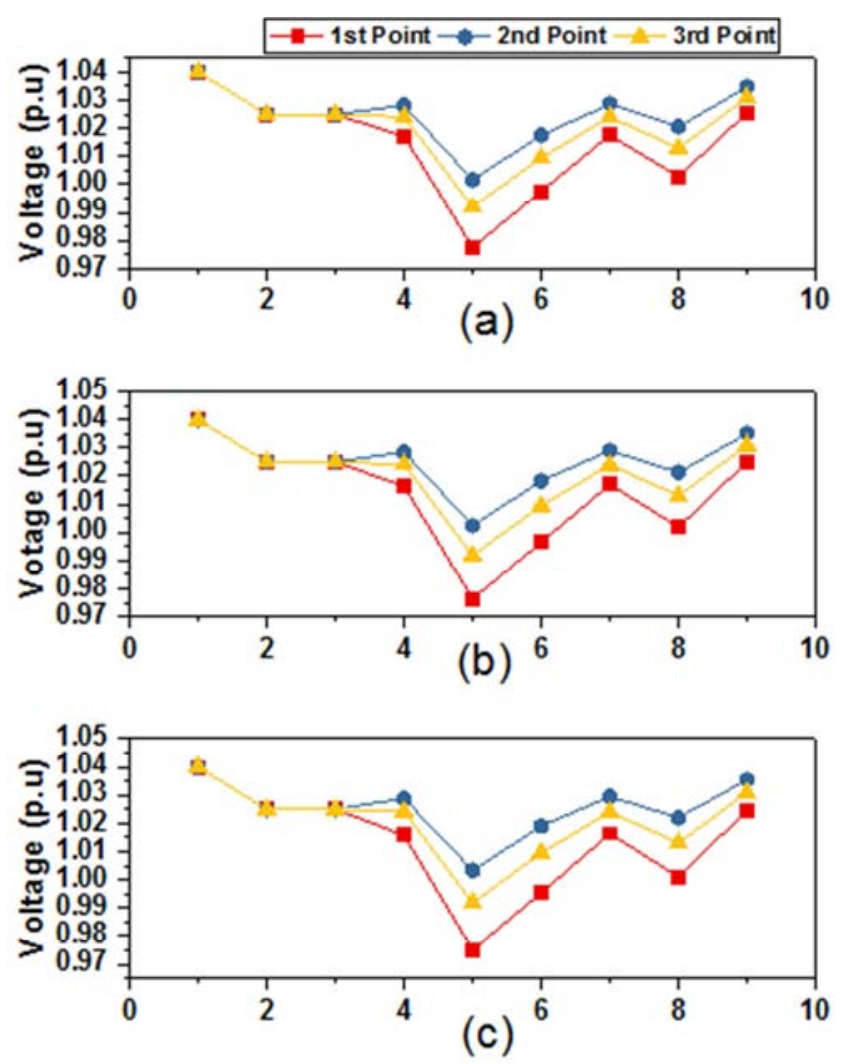

Bus No

Figure 4. Voltage magnitude at each bus, when $K_{k}=2.5,3, \& 3.5$.

Finally, the expected voltage magnitude (EVM) at each bus was calculated. The $2 m$ and $2 m+1$ scheme was compared with the base case (see Table 6), by looking the value of standard deviation, the results of the $2 \mathrm{~m}$ scheme is more deviate than $2 m+1$ scheme. So the value of EVM computed through the $2 m+1$ scheme is closer to the base case (see Fig. 5). Similarly, it applies to the line loading case (see Table 7), except those lines that are connected to the PV buses.

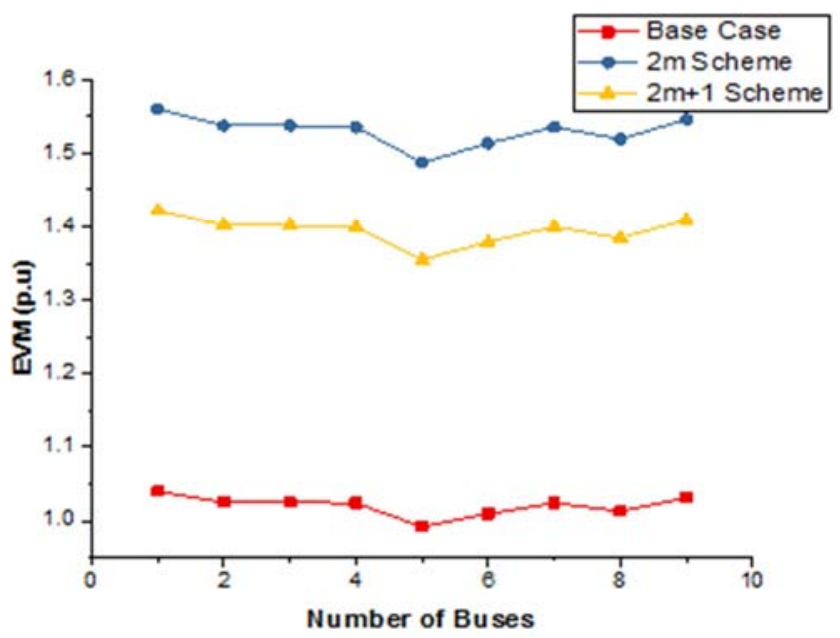

Figure 5. Comparison of EVM, when $K s=0 \& k_{k}=3$. 
Table 1. Comparison of ELL, when $K s=0 \& k_{k}=3$.

\begin{tabular}{llllll}
\hline $\begin{array}{l}\text { Line } \\
\#\end{array}$ & $\begin{array}{l}\text { Base } \\
\text { Case }\end{array}$ & $\begin{array}{l}\text { ELL } \\
\text { 2m }\end{array}$ & $\begin{array}{l}\text { ELL } \\
\mathbf{2 m + 1}\end{array}$ & $\begin{array}{l}\text { SD 2m \& } \\
\text { Base }\end{array}$ & $\begin{array}{l}\text { SD 2 m+1 \& } \\
\text { Base }\end{array}$ \\
\hline 1 & 16.160 & 16.393 & 18.064 & 0.116 & 0.952 \\
2 & 20.943 & 19.711 & 21.742 & 0.615 & 0.399 \\
3 & 19.699 & 9.1581 & 10.099 & 5.270 & 4.799 \\
4 & 9.1294 & 14.917 & 16.368 & 2.894 & 3.619 \\
5 & 14.700 & 10.468 & 11.544 & 2.115 & 1.578 \\
6 & 10.331 & 20.979 & 23.164 & 5.324 & 6.416 \\
\hline
\end{tabular}

\section{Conclusion}

In this paper Probabilistic power flow model used to study uncertainty with power system network. The proposed model based upon the point estimation method, $2 m$ and $2 m+1$ concentration scheme were used to evaluate the points of an input random variable. The program was written in DPL code by using the DIgSILENT Power Factory software. This model is used to create the uncertainty in load and compute the effect of this uncertainty in the power system network parameters. Especially, in this paper, expected voltage magnitude and expected line loading are considered for analysis purpose. From the analysis, it is shown that $2 m+1$ concentration scheme is more reliable than $2 m$ scheme.

The proposed model can address all possible uncertainties in loads and provide more reliable distribution of output data. Its implementation is investigated by using the P. M. Anderson 9-bus test system, and reliable corresponding results are shown in this paper. These results can be used for future planning, corrective action, and operation of electric power system.

\section{Acknowledgements}

First and foremost, I would like to thank my respectable teacher Mr Qi Bing and Mr Li Bin.

This work is supported by National Key Research and Development Program (2016YFB0901104), the National Natural Science Foundation of China (51307051), the Fundamental Research Funds for the Central Universities (2014ZP03, 2015ZD01) and the Science and Technology projects from State Grid Corporation.

\section{References}

[1] V. G. Dovì, F. Friedler, D. Huisingh, and J. J. Klemeš, "Cleaner energy for sustainable future," Journal of Cleaner Production, vol. 17, pp. 889-895, 2009.

[2] P. Basak, S. Chowdhury, S. H. nee Dey, and S. Chowdhury, "A literature review on integration of distributed energy resources in the perspective of control, protection and stability of microgrid," Renewable and Sustainable Energy Reviews, vol. 16, pp. 5545-5556, 2012.

[3] L. Tang, F. Wen, M. A. Salam, and S. P. Ang, "Transmission system planning considering integration of renewable energy resources," in 2015 IEEE PES Asia-Pacific Power and Energy Engineering Conference (APPEEC), 2015, pp. 1-5.
[4] X. Liang, "Emerging Power Quality Challenges Due to Integration of Renewable Energy Sources," IEEE Transactions on Industry Applications, vol. 53, pp. 855-866, 2017.

[5] S. Chun-Lien, "Probabilistic load-flow computation using point estimate method," IEEE Transactions on Power Systems, vol. 20, pp. 1843-1851, 2005.

[6] J. Usaola, "Probabilistic load flow in systems with wind generation," IET Generation, Transmission \& Distribution, vol. 3, pp. 1031-1041, 2009.

[7] M. Nassar and M. Salama, "Probabilistic power flow using novel wind and solar probabilistic models," in Power and Energy Society General Meeting (PESGM), 2016, 2016, pp. 15.

[8] F. Ni, P. Nguyen, J. Cobben, and J. Tang, "Application of non-intrusive polynomial chaos expansion in probabilistic power flow with truncated random variables," in Probabilistic Methods Applied to Power Systems (PMAPS), 2016 International Conference on, 2016, pp. 1-7.

[9] S. Peng, J. Tang, and W. Li, "Probabilistic Power Flow for AC/VSC-MTDC Hybrid Grids Considering Rank Correlation among Diverse Uncertainty Sources," IEEE Transactions on Power Systems, 2016.

[10] F. Ni, P. H. Nguyen, and J. F. Cobben, "Basis-Adaptive Sparse Polynomial Chaos Expansion for Probabilistic Power Flow," IEEE Transactions on Power Systems, vol. 32, pp. 694-704, 2017.

[11] M. Hajian, W. D. Rosehart, and H. Zareipour, "Probabilistic Power Flow by Monte Carlo Simulation With Latin Supercube Sampling," IEEE Transactions on Power Systems, vol. 28, pp. 1550-1559, 2013.

[12] X. Xu and Y. Gao, "Simulation on the active voltage balancing of series connected IGBTs by Monte Carlo analysis," in 2016 IEEE 11th Conference on Industrial Electronics and Applications (ICIEA), 2016, pp. 630-635.

[13] P. Jorgensen, J. Christensen, and J. Tande, "Probabilistic load flow calculation using Monte Carlo techniques for distribution network with wind turbines," in Harmonics and Quality of Power Proceedings, 1998. Proceedings. 8th International Conference On, 1998, pp. 1146-1151.

[14] P. Zhang and S. T. Lee, "Probabilistic load flow computation using the method of combined cumulants and Gram-Charlier expansion," IEEE transactions on power systems, vol. 19, pp. 676-682, 2004.

[15] T. Williams and C. Crawford, "Probabilistic load flow modeling comparing maximum entropy and GramCharlier probability density function reconstructions," IEEE Transactions on Power Systems, vol. 28, pp. 272280, 2013.

[16] Y. Chen, J. Wen, and S. Cheng, "Probabilistic load flow method based on Nataf transformation and Latin hypercube sampling," IEEE Transactions on Sustainable Energy, vol. 4, pp. 294-301, 2013.

[17] G. Verbic and C. A. Canizares, "Probabilistic optimal power flow in electricity markets based on a two-point estimate method," IEEE Transactions on Power Systems, vol. 21, pp. 1883-1893, 2006. 
[18] N. Soleimanpour and M. Mohammadi, "Probabilistic load flow by using nonparametric density estimators," IEEE Transactions on Power Systems, vol. 28, pp. 3747-3755, 2013.

[19] C. Long, M. E. A. Farrag, D. M. Hepburn, and C. Zhou, "Point Estimate Method for Voltage Unbalance Evaluation in Residential Distribution Networks with High Penetration of Small Wind Turbines," Energies, vol. 7, pp. 7717-7731, 2014.

[20] S. Mohammadi, B. Mozafari, and S. Solimani, "Optimal operation management of microgrids using the point estimate method and firefly algorithm while considering uncertainty,"
Turkish Journal of Electrical Engineering \& Computer Sciences, vol. 22, pp. 735-753, 2014.

[21] C.-L. Su, "A new probabilistic load flow method," in Power Engineering Society General Meeting, 2005. IEEE, 2005, pp. 389-394.

[22] P. M. Anderson and A. A. Fouad, Power system control and stability: John Wiley \& Sons, 2008.

[23] P. Sauer and M. Pai, "Power System Dynamics and Stability, Prentice-Hall," New Jersey, 1998. 